\title{
Comparison of the single-injection volar subcutaneous block and the two-injection dorsal block for digital anesthesia
}

\author{
V. Monsef Kasmaei ${ }^{1^{*}}$, M. T. Talebian ${ }^{2}$, A. Shams Akhtari ${ }^{3}$, Sh. Keykhah ${ }^{4}$ \\ ${ }^{1}$ Guilan Road Trauma Center, Guilan University of Medical Sciences, Rasht, Iran; *Corresponding Author: Vmonsef@yahoo.com \\ ${ }^{2}$ Emergency Department, Tehran University of Medical Sciences, Tehran, Iran \\ ${ }^{3}$ Emergency Department, Shahid Beheshti University of Medical Sciences, Tehran, Iran \\ ${ }^{4}$ Emergency Department, Mashhad University of Medical Sciences, Mashhad, Iran
}

Received 6 June 2013; revised 7 July 2013; accepted 8 August 2013

Copyright (C) 2013 V. Monsef Kasmaei et al. This is an open access article distributed under the Creative Commons Attribution License, which permits unrestricted use, distribution, and reproduction in any medium, provided the original work is properly cited.

\section{ABSTRACT}

Objectives: Local digital nerve blockade is frequently used in many trauma cases. Two commonly used techniques of digital nerve block with local anesthetic are the two-injection dorsal technique and the single-injection volar subcutaneous technique. In this study we compare various parameters of the single-injection volar subcutaneous block and the two-injection dorsal block. Pain score, amount of injected anesthetic, time of effect onset, patients' and physicians' satisfaction scores in each injection technique was compared. Methods: 128 participating patients were randomly divided into two equal experimental groups. Two-percentage Lidocaine was used as an anesthetic agent. Doses of $\mathbf{1 . 8}$ and $3-4 \mathrm{ml}$ were used in the single-injection subcutaneous block and the two-injection dorsal block groups, respectively. Following injections, the patients were asked to score their discomfort experience on a standard visual analog scale of $\mathbf{0}$ (no pain) to 10 (most pain imaginable). They also were asked to score the rate of their satisfaction from 1 (no satisfaction) to 5 (most satisfaction). The onset of effect was determined using the pinprick test. Results: Our results demonstrate that the two-injection dorsal block technique imposes more pain but the pain score difference was not statistically significant. Both patients and physicians were more comfortable with the single-injection subcutaneous digital block method. This satisfaction difference was statistically significant. Conclusions: The single-injection method is more efficient and the patients were more pleased. The advantages of this method are its safety, user friendly, need of lower amount of anesthetic drug and its easiness to teach and learn.

Keywords: Hand Surgery; Volar Subcutaneous Block; Two-Injection Dorsal Block

\section{INTRODUCTION}

Local digital nerve blockade is frequently used in many trauma cases. Like other nerve blockade methods, digital nerve supplying anatomy has led to the development of many different techniques of digital nerve blockade. The classic method of two-injection dorsal digital block, first proposed by Harris and Broun [1], is widely used (Figure 1). A new method described by Harbison for more than ten years ago is the subcutaneous volar block [2]. The volar approach is an alternative to the traditional two-injection dorsal block and typically involves only a single injection. There are two basic variations of the volar blockade. Chui (1990) used the transthecal digital block method in which anesthetic drug is delivered to finger through volar single-injection into flexor tendon sheath acts as a conduit [3]. However, previous studies showed that this method is not much appropriate due to the unpleasant feeling caused by the needle entrance into the tendon sheath [4].

Single injection subcutaneous digital block is another type of finger blockade which was first proposed by Harbison [5]. Lower potential risks of damage to nerves and capillaries of finger with less pain and more applicability than the other methods are expected [6] (Figure 2).

To our knowledge, the single-injection volar subcutaneous block proposed by Harbison has not been com- 


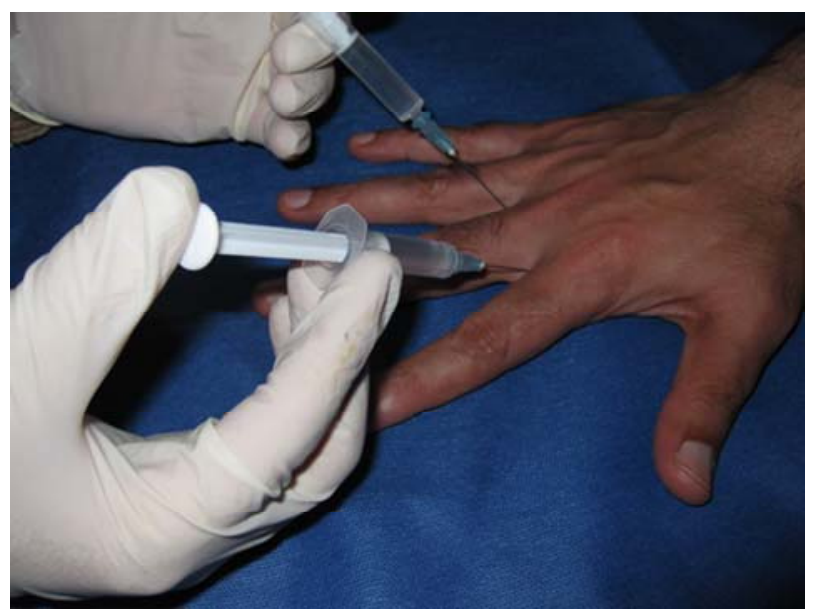

Figure 1. The classic method of two-injection dorsal digital block in finger.

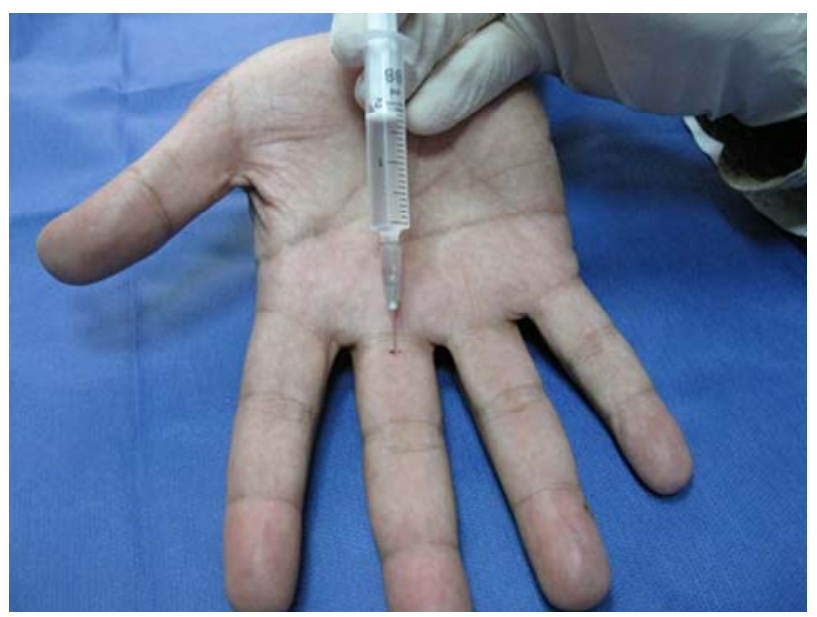

Figure 2. Single-injection volar subcutaneous digital block in finger.

pared with a traditional two-injection dorsal block approach in a controlled, prospective designed study with relative large sample size.

This aim of our study is to compare the two digital block methods with respect to pain score, time of anesthesia, and patients and physicians' satisfaction scores about the injection technique.

\section{METHODS}

All of this study design and steps was accepted by Research Ethics and Review of Tehran University of Medical sciences. Samples consist of all volunteer patients requiring digital block, visiting the emergency department of Imam Khomeini hospital (Tehran, Iran) from July 1, 2007 to January 1, 2008.

All the patients had finger-damaged and were above 18 years old of age. Exclusion criteria included a history of allergy to the local anesthetic drugs, evidence of an active local infection in the injection area, age below 18 years old, suffering from detected coalescence disorder, preexisting vascular insufficiency, severe peripheral vascular disease, history of previous digital replantation, previous participation of subject in this study and presence of the lesion was in the dorsal proximal phalanx.

Informed consent was obtained from each subject. All stages of this study were in accordance with Helsinki Declaration on Patient Safety in Anesthesiology. Patients' confidential data have been protected, surgeries and medical actions have been performed based on the written consent of patients. Furthermore, all experimental procedures have been conducted solely by competent technicians under the supervision of proficient physicians. In all medical operations painful activities have been performed after full anesthesia confirmation.

128 subjects were randomly divided into two equal main experimental groups using block randomization method. Fingers of the first and the second groups' members were anesthetized using the single injection subcutaneous digital block method and common two injection dorsal digital block method, respectively. We used $2 \%$ lidocaine as an anesthetic agent and doses of 1.8 and 3 $4 \mathrm{ml}$ were used in the single-injection subcutaneous block and the two-injection dorsal block groups, respectively.

In the single-injection subcutaneous block, the needle was inserted 3 to $4 \mathrm{~mm}$, directed toward the base of the digit. Subcutaneous injection of $1.8 \mathrm{ml}$ of anesthetic drug was performed through the volar technique just deep to the skin in the midline at the level of the proximal flexion crease of the damaged finger. All injections were performed carefully to ensure that the volar injection was neither intradermal (too superficial) nor intrathecal (too deep).

After each block, the subjects were asked to score their discomfort experienced on a standard Visual Analog Scale (VAS) of 0 (no pain) to 10 (mostpain imaginable). This assessment was performed by a blinded evaluator (blinded study).The interval between the time of injection and beginning of finger anesthesia was determined using the pinprick test.

After finishing the surgery and a minimum of 30-minrecovery period, the subjects were asked to score the rate of their satisfaction from 1 (no satisfaction) to 5 (most satisfaction). The other data including the satisfaction scores of physician, gender and age of subjects were also imported into the study checklists and saved into computers for statistical analyses.

\section{STATISTICAL ANALYSIS}

The obtained data were categorized and sorted as Code Sheets and Master Sheets and then imported into a PC. Statistical Package for the Social Sciences (SPSS) version 15 was used for analysis of the data. 
Independent sample test (t-test) was used to analyze and compare the mean age of the subjects and mean age of the groups. T-tests were also used to compare the mean pain scores and intervals between the injections and onset of finger anesthesia between the experimental groups.

Chi-square tests were used to examine the relationship between gender and the two variables of patients and physicians satisfaction levels in the experimental groups regarding the two digital anesthetic methods.

The significance level of $\mathrm{p}<0.05$ was applied for all of the statistical tests.

\section{RESULTS}

The research sample consists of 128 patients; 18 female and 110 male. Their average age was 28.9 years old. Statistical analyses have not shown any significant difference between subjects in terms of gender, average age, two groups in terms of the two digital anesthetic meth- ods ( $p>0.09$ ). Also $\mathrm{T}$ test showed no significant difference between the two groups in terms of the VAS, (Table 1).

Chi-square test, showed significant difference between the patients' satisfaction in the two groups ( $p<0.001)$. In the single-injection subcutaneous digital block method the mean pain score of patients' satisfaction was more than the other group (Table 2).

The Chi square test, examined the difference between the physicians' satisfaction levels in the two groups, showed a significant difference $(\mathrm{p}<0.006)$. Physicians' satisfaction in the first group - treated with the singleinjection subcutaneous digital block method-was more than the other group (Table 3 ).

Our data analysis relieved that the time of injection and beginning of anesthesia showed statistically significant difference between the two groups $(\mathrm{p}<0.001)$. The interval in the single-injection was more than the two-injection group (Table 4).

Table 1. The pain score of the two groups of patients.

\begin{tabular}{ccccc}
\hline $\begin{array}{c}\text { p-Value Based on } \\
\text { Independent Samples t-Test }\end{array}$ & Standard Deviation & Average & Frequency & Group \\
\hline \multirow{2}{*}{0.16} & 1.77 & 4.18 & 64 & Single-Injection Volar \\
& 2.15 & 4.67 & 64 & Two-Injection Dorsal \\
\hline
\end{tabular}

Table 2. The patients' satisfaction scores in the two groups.

\begin{tabular}{|c|c|c|c|c|c|c|c|}
\hline \multirow{3}{*}{$\begin{array}{l}\text { p-Value } \\
\text { Based on } \\
\text { Chi-Square }\end{array}$} & \multirow{2}{*}{\multicolumn{2}{|c|}{ Sum }} & \multicolumn{4}{|c|}{ Patients' Satisfaction } & \multirow{3}{*}{ Group } \\
\hline & & & \multicolumn{2}{|c|}{ Dissatisfaction } & \multicolumn{2}{|c|}{ Satisfaction } & \\
\hline & Percentage & Frequency & Percentage & Frequency & Percentage & Frequency & \\
\hline \multirow{2}{*}{0.02} & 100 & 64 & 34.4 & 22 & 65.6 & 42 & Single-Injection Volar \\
\hline & 100 & 64 & 62.5 & 40 & 37.5 & 24 & Two-Injection Dorsal \\
\hline
\end{tabular}

Table 3. The physicians' satisfaction levels in the two groups.

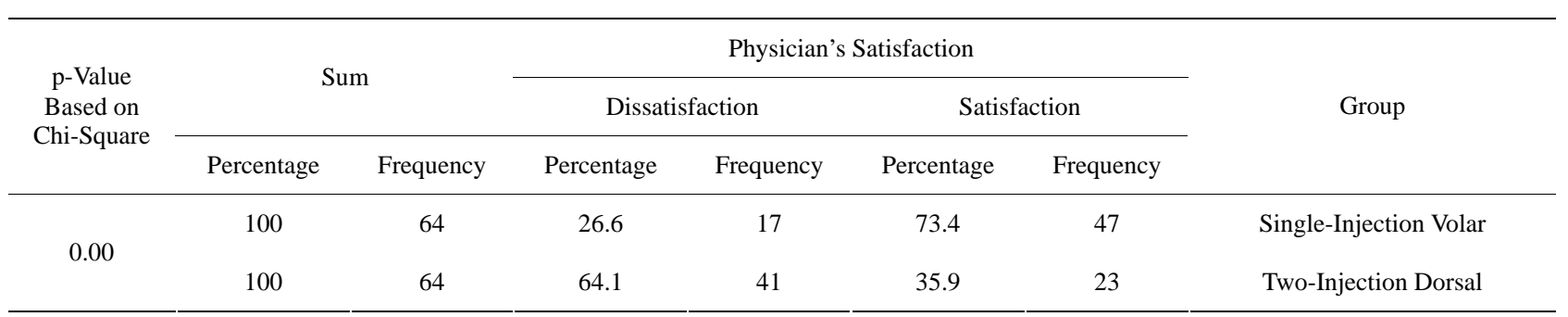

Table 4. The interval between the time of injection and beginning of the anesthesia in groups.

\begin{tabular}{ccccc}
\hline $\begin{array}{c}\text { p-value Based on Independent } \\
\text { Samples t-Test }\end{array}$ & Standard Deviation & Time Interval (s) & Frequency & Group \\
\hline \multirow{2}{*}{0.001} & 40.87 & 216.56 & 64 & Single-Injection Volar \\
& 31.60 & 194.06 & 64 & Two-Injection Dorsal \\
\hline
\end{tabular}




\section{DISCUSSION}

We compared the single-injection volar subcutaneous block and the two-injection dorsal block for anesthesia of the finger. Our result showed no significant difference between the two groups in terms of the different surgical operated finger's type and these two methods are equally effective in the index, small and thumb fingers as they are in the middle and ring fingers of both left and right hands.

Our results also demonstrate that the traditional method imposes more pain. But the pain score difference was not statistically significant. These results confirm the findings of Bashir's research conducted on 30 patients. Their results revealed that volar and dorsal block methods had achieved $100 \%$ and $80 \%$ success, respectively [2]. Furthermore Williams, Jason and Lalonde (2004) conducted a research in Canada which showed that the pain score difference between the two methods is not statistically significant, but 22 out of the 27 patients treated with both blocks preferred the single-injection subcutaneous digital block method to the common digital method [6].

In our research patients and physicians were also more satisfied with the single-injection subcutaneous digital block method. This satisfaction difference was statistically significant. There were some probable reasons to explain why patients were more satisfied with the single-injection subcutaneous digital block method while there was no significant difference between two methods' mean pain scores. First, the subjects were not adequate to reveal the probable difference. Second, the scale of 0 (no pain) to 10 (the most serious pain) does not likely have enough sensitivity and detailed range to cover all the experienced pain or distinguish the little difference between different intensities of pain. Hence our patients were asked about their satisfaction scores in terms of pain score. On the other hand, patients, regardless of their pain score, most likely prefer single-injection to twoinjection method and receiving two needle insertions in their fingers.

The findings of the present study demonstrate that the mean interval between the times of injections and beginning of anesthesia in single-injection, as compared to the two-injection method, has more values. This could be because of the relative far distance between the finger's middle line (the area of injection in single-injection me- thod) and the finger's nerves [7]. Although for saving the time and for less discomfort of patient, it is better to reduce the interval, but we recommend the application of single-injection method instead of the traditional method because this method is easy to teach and learn. Due to the fact that, in this method, injection is performed in the finger's middle line, there is less risk of trauma in finger's nerves and capillaries. Finally, the most important advantage of the single-injection subcutaneous digital block method, as compared to two-injection dorsal digital block method, is the lower dosage of the anestheticdrug for achieving full digital anesthesia (1.8 and 3 - 4 $\mathrm{mm}$ in the first and second methods respectively).

\section{REFERENCES}

[1] Braun, H. and Harris, M.L. (1924) Operations on the extremities. In: Braun, H. and Harris, M.L., Eds., Local Anesthesia: Its Scientific Basis and Practical Use, 2nd Edition, Lea \& Febiger, New York, 1924, 366-367.

[2] Bashir, M.M., Khan, F.A., Afzal, S. and Khan, B.A. (2008) Comparison of traditional two injections dorsal digital block with volar block. Journal of College of Physicians and Surgeons Pakistan, 18, 768-770.

[3] Chui, D.T.W. (1990) Transthecal digital block: Flexor tendon sheath used for anesthetic infusion. Journal of Hand Surgery (American Volume), 15, 471. http://dx.doi.org/10.1016/0363-5023(90)90063-W

[4] Keramidas, E.G., Rodopoulou, S.G., Tsoutsos, D., Miller, G. and Ioannovich, J. (2004) Comparisonof transthecal digital block and traditional digital block for anesthesia of the finger. Journal of Plastic and Reconstructive Surgery, 114, 1131-1134.

[5] Harbison, S. (1991) Transthecal digital block: Flexor tendon sheath used for anesthetic infusion (Letter). Journal of Hand Surgery (American Volume), 16, 957. http://dx.doi.org/10.1016/S0363-5023(10)80172-8

[6] Williams, J. and Lalonde, D. (2006) Randomized comprison of the single-injection volar subcutaneous block and the two-injection dorsal block for digital anesthesia. Journal of Plastic and Reconstructive Surgery, 118, 11951200.

http://dx.doi.org/10.1097/01.prs.0000237016.00941.96

[7] O’Rahilly, R. and Müller, F. Carpenter and Swenson (2008) Basic human anatomy: A regional study of human structure. W. B. Saunders Co., Philadelphia. 